\title{
A THEOREM OF DIFFERENTIAL MAPPINGS OF RIEMANN SURFACES
}

\author{
PEICHU HU \\ Department of Mathematics, Shandong University \\ Jinan, Shandong, P. R. China \\ MINGZE YANG \\ Department of Mathematics, University of Saskatchewan \\ Saskatoon, Sask. Canada S7N 0W0 \\ (Received January 9, 1992 and in revised form March 23, 1992)
}

\begin{abstract}
In this paper, we have extended S.S. Chern's second basic theorem about holomorphic mapping between two Riemann surfaces to more general case, and also obtained two similar results.
\end{abstract}

KEY WORDS AND PHRASES: Riemann surfaces, differential mapping, meromorphic functions, critical points, integral form.

1980 AMS SUBJECT CLASSIFICATION CODES: 30F99, 30D20, 30D30.

\section{INTRODUCTION.}

Since Nevanlinna founded value distribution theory of meromorphic functions in 1925 , this theory has achieved many results until now. In the case of several complex variable analysis, $\mathrm{H}$. Kneser [8] (1938), H.Cartan [4] (1932), L.Ahlfors [1] (1941), Levine-Chern [10], [6] (1960), BottChern [2] (1965), W.Stoll [11], [12], Carlson-Griffiths-King [3], [7] had' obtained different forms of Nevanlinna theory. In 1960, S.S. Chern [5] used L.Ahlfors' method and obtained two basic theorems and inequality of deficient values about holomorphic mapping between two Riemann surfaces.

Let $M$ be a compact Riemann surface, $G$ be a Hermitian metric of $M$ which has constant Gauss curvature $K$. Let $\Omega$ be the volume element of $G$. For every $a \in M$, Chern has proved that there exsits a real function $U_{a}$ which is $C^{\infty}$ on $M-\{a\}$, and satisfies:

$$
\lambda_{a}=\Omega
$$

where $\lambda_{a}=\frac{1}{2 \pi} d^{c} U_{a}, d^{c}=i(\bar{\partial}-\partial)$. If $z$ is a local coordinate function on the neighbour $U$ of $a$, such that $z(a)=0$, then $U_{a}(z)+\log |z|$ is $C^{\infty}$ on $U$. In [5], Chern proves the following theorems.

THEOREM A. Let $D$ be a compact differentiable, orientable domain bounded by a sectiuonally smooth curve $\partial D, f: D \longmapsto M$ is a differential mapping, if $a \in M$ such that, $f^{-1}\{a\} \cap \partial D=\emptyset$, and $f^{-1}\{a\}$ is a finite set of points, then we have: 


$$
n(D, a)+\int_{\partial D} f^{*} \lambda_{a}=v(D)
$$

where $v(D)=\frac{1}{A} \int_{D} f^{*} \Omega\left(A=\int_{M} \Omega\right), n(D, a)$ is the counting function of $f$.

THEOREM B. Let $D$ be compact Riemann surface with smooth boundary $\partial D, f: D \longmapsto M$ is a holomorphic mapping, then

$$
\chi(D)-\frac{1}{2 \pi} \int_{\partial D} K+n_{1}(D)=\chi(M) v(D),
$$

where $\chi(D)$ and $\chi(M)$ are Euler's characteristics of $D$ and $M$ respectively, $n_{1}(D)$ is the stationary index of $f$ in $D$.

For the case of holomorphic mappings, S.S.Chern gave the integral form of theorem A and theorem $\mathrm{B}$, and also proved the ralation inequlity of deficient values. In this paper, we replace $f$ by differential mapping, and also get similar results. We have the following main result.

THEOREM 1. Let $D$ be compact Riemann surface with smooth boundary $\partial D$, if $f: D \longmapsto M$ is a differential mapping, and if the critical points of $f$ are all isolated points, $f$ is orientation-preserving except critical points. Then we also have equality (3).

\section{The Proof of Theorem 1.}

By using local coordinate $z=x+y i$, we have $G=g d z d \bar{z}=g\left(d x^{2}+d y^{2}\right)$, and $\Omega=\frac{1}{2} g d z \wedge d \bar{z}=$ $g d x \wedge d y$, where $g$ is a positive function which belongs to $C^{\infty}$.

Lemma 1 (Gauss-Bonnet formula). If $\Delta$ is a compact subset of $M$ with smooth boundary $\partial \Delta$, let $K=K_{g} d s$ be the curvature form of $\partial \Delta$ about $G$, where $K_{g}$ is the curvature of $\partial \Delta$, then

$$
2 \pi \chi(\Delta)-\int_{\partial \Delta} K=\int_{\Delta} K \Omega
$$

especially, $\chi(M)=\frac{1}{2 \pi} \int_{M} K \Omega$.

Now we define stationary index $n_{1}(D)$ of differential mapping of $f: D \longmapsto M$ as the following:

We suppose that $\alpha_{1}, \alpha_{2}, \ldots, \alpha_{n}$ are the critical points of $f$ in $D-\partial D$ (i.e. $d f\left(\alpha_{\jmath}\right)=0, j=$ $1,2, \ldots, n)$. Because $f$ is orientation-perserving (except the critical points), then the metric of $G$ on $M$ can induce Hermitian metric $f^{*} G$ on $D-\left\{\alpha_{1}, \ldots, \alpha_{n}\right\}, f: D-\left\{\alpha_{1}, \ldots, \alpha_{n}\right\} \longmapsto M$ is local isometry mapping, so $f^{*}(K \Omega)$ is equal to the product of Gauss curvature of $f^{*} G$ on $D-\left\{\alpha_{1}, \ldots, \alpha_{n}\right\}$ and volume element of $f^{*} G$. We suppose that $z_{1}$ is the local coordinate function in the neigbohour of $\alpha_{\imath}$ such that $z_{\imath}\left(\alpha_{\imath}\right)=0, W,=\left\{\left|z_{\jmath}\right|<\epsilon\right\}, W=\bigcup_{j=1}^{n} W_{j}$. We use $K$ denote the geodesio curvature form of $\partial W$ about $f^{*} G$.

Now we define stationary index $I_{\alpha_{i}}$ of $f$ at $\alpha_{i}$ as the following:

$$
I_{\alpha_{i}}=\lim _{\epsilon \rightarrow 0} \frac{1}{2 \pi} \int_{\partial W_{\imath}} K-1,
$$

and call $n_{1}(D)=\sum_{\jmath=1}^{n} I_{\alpha}$, is the stationary index of $f$ in $D$. We apply the lemma 1 to the metric of $f^{*} G$ in $D-W$, then $2 \pi \chi(D-M)-\int_{\partial D} K+\int_{\partial W} K=\int_{D-M} f^{*}(K \Omega)$, where the orientation of $\partial W$ is induced by the orientation of $W$. Clearly, $\chi(D-M)=\chi(D)-n$, and $\lim _{\epsilon \rightarrow 0} \int_{D-W} f^{*}(K \Omega)=$ $\int_{D} f^{*}(K \Omega)$. So

$$
\begin{aligned}
\int_{D} f^{*}\left(K^{\prime} \Omega\right) & =2 \pi \chi(D)-\int_{\partial D} K+2 \pi \lim _{\epsilon \rightarrow 0} \sum_{j=1}^{n}\left(\frac{1}{2 \pi} \int_{\partial W,} K-1\right) \\
& =2 \pi \chi(D)-\int_{\partial D} K+2 \pi n_{1}(D) .
\end{aligned}
$$

We notice that $K$ is constant, then apply Gauss-Bonnet formula, we have: 


$$
\lambda(M)=\frac{1}{2 \pi} \int_{M} K \Omega=\frac{K}{2 \pi} \int_{M} \Omega=\frac{K}{2 \pi} A .
$$

So we have

We are done.

$$
\frac{1}{2 \pi} \int_{D} f^{*}(K \Omega)=\frac{K A}{2 \pi} \frac{1}{A} \int_{D} f^{*} \Omega=\chi(M) v(D)
$$

\section{Integral Form of Theorem 1.}

Let $V$ be a open Riemann surface. Suppose that $V$ has an infinite harmonic exhaustion function $\tau$ [13]. We also suppose that $f: V \longmapsto M$ is a differential mapping, and all critical points are isolated points, $f$ is orientation-preserving (except critical points), if $\varphi$ is one-form on $V$, let ${ }^{*} \varphi$ be the conjugate one-form.

We let $V[r]=\{p \mid \tau(p) \leq r\}$, if $r$ is not the critical values of $\tau$, then $V[r]$ is compact subset in $V$ with smooth boundary. Let $n(r, a)=n(V[r], a), v(r)=v(V[r]), \chi(r)=\chi(V[r]), n_{1}(r)=n_{1}(V[r])$. For $f$, we use theorem 1 , we conclude

$$
\chi(r)-\frac{1}{2 \pi} \int_{\partial V[r]} K+n_{1}(r)=\chi(M) v(r)
$$

where $K$ is geodesio curvature form of $\partial V[r]$ about $f^{*} G$. We can introduce function $h$ such that, $f^{*} \Omega=h d \tau \wedge \star d \tau$ on $V-V[r(\tau)]$, because $f$ is orientation-persveing, so $f^{*} \Omega$ and $V$ have the same orientation, clearly, $d \tau \wedge \star d \tau$ and $V$ have the same orientation, so $h$ is nonnegative function, and $K=\frac{1}{2} d^{c} \log h$, then we have $\int_{\partial V[r]} K=\frac{1}{2} \int_{\partial V[r]} d^{c} \log (h)$. According to [13], we can use special coordinate function $\sigma=\tau+i \rho$, so

$$
d^{c} \log (h)=-\frac{\partial \log (h)}{\partial \rho} d \tau+\frac{\partial \log (h)}{\partial \tau} d \rho
$$

and

$$
\frac{1}{2} \int_{\partial V[r]} d^{c} \log (h)=\frac{1}{2} \int_{\partial V[r]} \frac{\partial \log (h)}{\partial \tau} d \rho=\frac{\partial}{\partial r}\left(\frac{1}{2} \int_{\partial V[r]} \log (h) \star d \tau\right) .
$$

By using the method which we deal with holomorphic functions, we can introduce the following functions: $E(r)=\int_{r_{0}}^{r} x(t) d t, N_{1}(r)=\int_{r_{0}}^{r} n_{1}(t) d t$, and $T(r)=\int_{r_{0}}^{r} v(t) d t$, where $r>r_{0} \geq r(\tau)$. Because of (5), we have

$$
E(r)+N_{1}(r)-\left.\frac{1}{4 \pi} \int_{\partial V[t]}(\log (h)) \star d \tau\right|_{r_{0}} ^{r}=\chi(M) T(r) .
$$

This is the integral form of theorem 1.

\section{References}

[1] L. Ahlfors, The Theory of Meromorphic Curves, Acta Soc. Sci., Fenn. Nova Ser. A 3 (4) (1941), 171-183.

[2] R. Bott and S.S.Chern, Hermitian Vector Bundles and Equidistribution of the Zeros of Their Holomorphic Sections, Acta Math., 114 (1965), 71-112.

[3] J.Carson and P.Griffiths, Defect Relation for Equidimensional Holomorphic Mappings Between Algebriac Varieties, Ann. of Math., 95 (1972), 557-584. 
[4] H.Cartan, Sur les zeros des combinaisons lineares de p fonctions holomorphes donnees, Mathematica (cluj), 7 (1933), 80-103.

[5] S.S.Chern, Complex Analytic Mappings of Riemann Surfaces I, Amer. J. Math., 82 (1960), 323-337.

[6] S.S.Chern, The Integrated Form of the First Main Theorem for Complex Analytic Mappings in Several Variables. Ann. of Math., (2) 71 (1960), 536-551.

[7] P. Griffiths and J. King, Nevanlinna Theory and Holomorphic Mappings Between Algebraic Varieties, Acta. Math., 130 (1973), 145-220.

[8] H. Kneser, Zur Theorie der gebrochenen Funktionen mehrerer, Veranderlichen. Jber. Deutsch. Math. Verein., 48 (1938), 1-28.

[9] R. Nevanlinna, Zur Theorie der meromorphen Funktionen, Acta Math., 46 (1925), 1-99.

[10] H. Levine, A Theorem on Holomorphic Mappings into Complex Projective Space, Ann. of Math., (2) 71 (1960), 529-535.

[11] W.Stoll, A General first main theorem of value distribution, Acta Math., 118 (1967), 111-191.

[12] W. Stoll, Die beiden Hauptsatze der Wertverteilungstheorie bei Funktionen mehrerer komplexen Veranderlichen I, Acta Math., 90 (1953), 1-115, II Acta Math., 92 (1954), 55-169.

[13] H. Wu, The equidistribution theory of holomorphic curves, Ann. of Math. Studies, 64, Princeton Univ. Press, Princeton, NJ, (1970). 


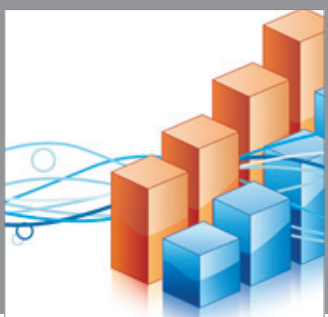

Advances in

Operations Research

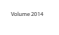

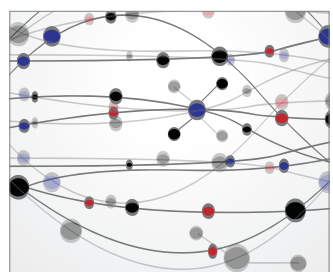

\section{The Scientific} World Journal
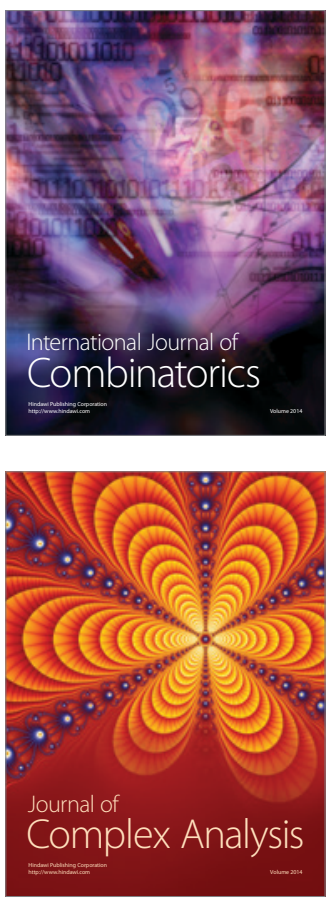

International Journal of

Mathematics and

Mathematical

Sciences
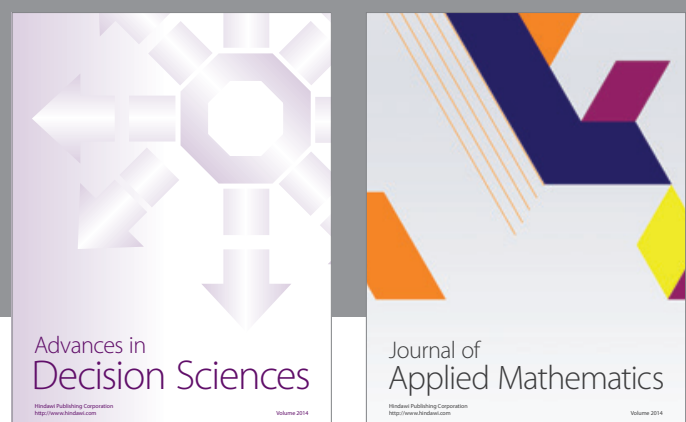

Journal of

Applied Mathematics
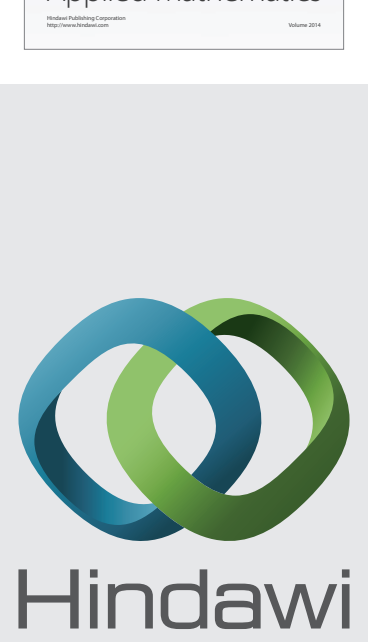

Submit your manuscripts at http://www.hindawi.com
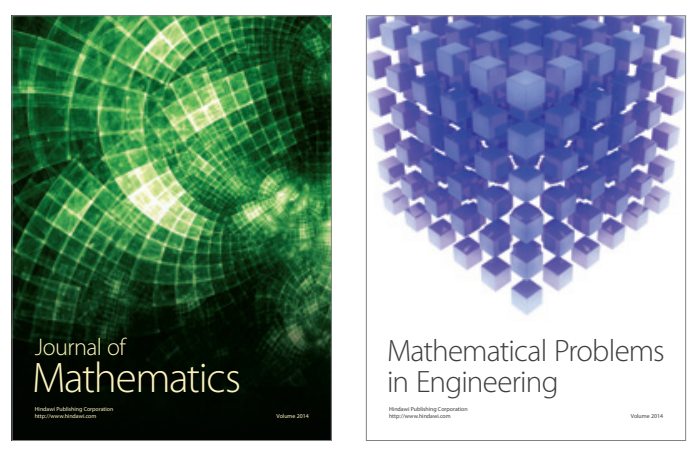

Mathematical Problems in Engineering
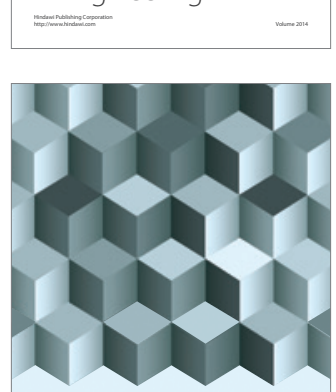

Journal of

Function Spaces
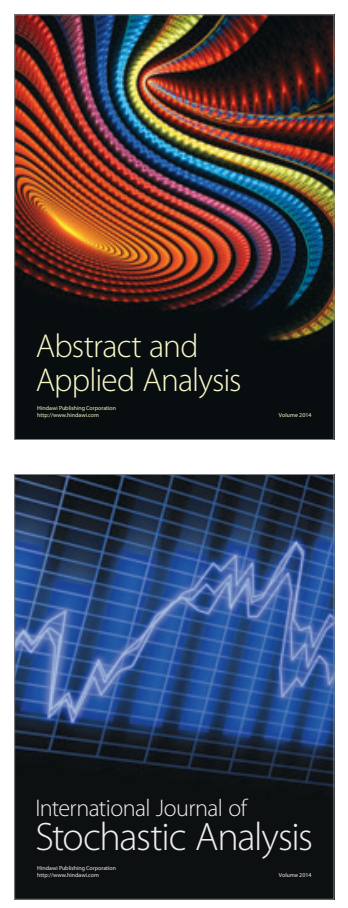

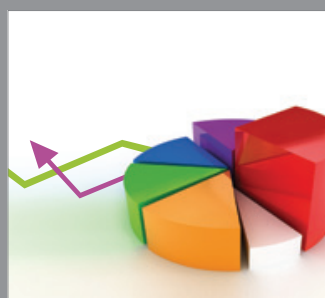

ournal of

Probability and Statistics

Promensencen
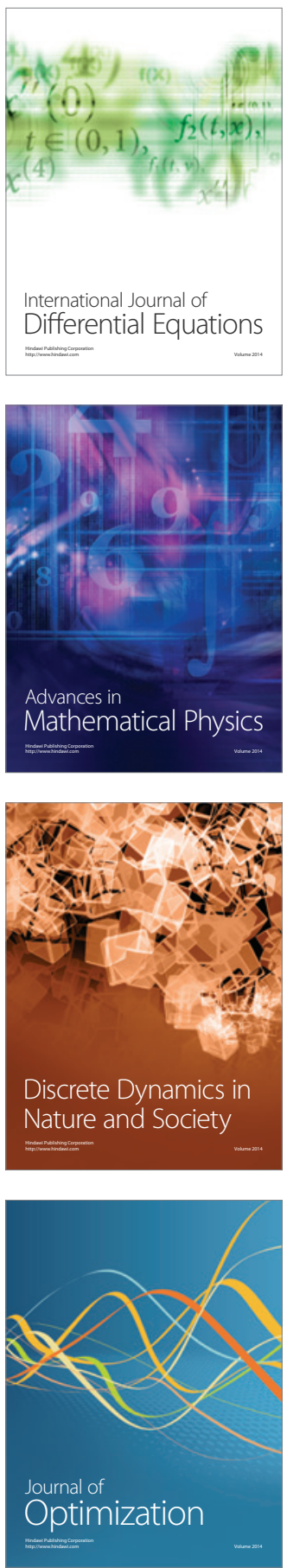